\title{
Side matters: potential mechanisms underlying dogs' performance in a social eavesdropping paradigm
}

Marie Nitzschnera, Juliane Kaminskib, Alicia Melisc, Michael Tomaselloa

\section{Abstract}

Social eavesdropping is the gathering of information by observing interactions between other individuals. Previous studies have claimed that dogs, Canis familiaris, are able to use information obtained via social eavesdropping, that is, preferring a generous over a selfish human donor. However, in these studies the side was constant between the demonstrations and the dogs' choices, not controlling for potential location biases. In the crucial control condition of our experiments, the donors swapped places in half of the trials before the dogs chose. We found that first choice behaviour as well as the time dogs interacted with the generous donor were influenced by location (side). In a second experiment the subject's owner interacted with the two donors. Again, the result of the side control revealed that the critical factor was location (side) not person. The results of these experiments provide no evidence for social eavesdropping in dogs and show the importance of critical control conditions.

The ability to use information about unknown individuals on the basis of third-party interactions is widespread in the animal kingdom (birds: Amy \& Leboucher, 2007; fish: Bshary and Grutter, 2006, Danchin et al., 2004 and Paz-y-Miño C et al., 2004; for a review of other species see Valone, 2007). In most species, such skills are confined to fighting or mating contexts and therefore are probably highly constrained (Subiaul, Vonk, Okamoto-Barth, \& Barth, 2008).

In contrast, in humans the assessment of others based on indirect experience is a highly flexible ability that is considered to be a key component in human cooperation (Nowak and Sigmund, 1998, Nowak and Sigmund, 2005 and Wedekind and Milinski, 2000). There is also some evidence that our closest relatives, the chimpanzees, Pan troglodytes, use information gathered by witnessing interactions between others (Russell, Call, \& Dunbar, 2008). More precisely, the chimpanzees in that study observed interactions of a beggar with a food-sharing experimenter versus a food-withholding experimenter and afterwards displayed a preference for the food-sharing experimenter. However, in another study, chimpanzees did not show a spontaneous preference for a 'generous' donor (Subiaul et al., 2008). Given the inconsistent results it remains unclear whether chimpanzees are flexibly able to gather information about humans' food-sharing behaviour via third-party interactions.

Several studies provide some evidence that dogs' cognitive skills in some domains seem to be more flexible than those of species more closely related to humans (Bräuer et al., 2006 and Hare et al., 2002). Dogs are social-living animals and have lived among humans for at least 15000 years (Savolainen et al., 2002 and 
Vilà et al., 1997). The findings of the latest study even suggest an onset of domestication in Europe up to 32100 years ago (Thalmann et al., 2013). During this time they have developed a number of outstanding sociocognitive skills, which have enabled them to interact and communicate with humans (Miklósi, Topál, \& Csányi, 2004).

Since dogs rely on humans to provide them with food (Clutton-Brock, 1995 and Coppinger and Coppinger, 2002), it should also be an advantage for a dog to be able to assess humans' food-sharing tendencies via observation. Indeed, two recent studies have shown that dogs seem to use information about humans' food-sharing tendencies after having observed several interactions between a food-giving (generous) donor and food-withholding (selfish) donor and an unknown human beggar. Kundey et al. (2010) found that dogs chose the demonstrator who gave food to a human recipient more often than the withholding demonstrator. However, the subjects in this study also favoured the human who 'gave' food to a wooden box over a 'selfish' human, raising the possibility that rather than assessing the experimenters' food-sharing behaviour based on an interaction they had witnessed, the dogs simply associated food with one but not with the other donor. In a similar study, Marshall-Pescini, Passalacqua, Ferrario, Valsecchi, and PratoPrevide (2011) also showed that dogs preferred the food-giving over the food-withholding experimenter. Additionally, they conducted a ghost control, in which no beggar was present but the demonstrators performed the same actions as in the experimental condition. In this control condition, dogs did not prefer one over the other experimenter. This finding shows that the dogs did not prefer the food-sharing experimenter because of her specific behaviour but took the actual interactions between the beggar and the donor into account. However, in this ghost control no food transfer took place, which could be the crucial difference between the two different beggar-donor interactions in the experimental versus the ghost control condition. Therefore, it is possible that dogs simply preferred the side where they saw a food transfer during the beggar-donor interactions in the experimental condition (since the positions of the experimenters were not altered between the observation and test choosing phase). With this method it is not possible to rule out location bias as a potential factor influencing the dogs' performance. In contrast to the two studies highlighted, which yielded positive findings on dogs' social-eavesdropping abilities, another recent study found no evidence for a flexible use of information gathered via observation of third-party interactions in dogs (Nitzschner, Melis, Kaminski, \& Tomasello, 2012). In that study, subjects preferred a nice experimenter, who played with them, over an ignoring experimenter, who ignored them completely, after they had had direct experiences with them both, but not if they only witnessed interactions between the two experimenters and another dog well known to them. However, in that study no food was involved and no local cues were provided, minimizing the possibility of forming associations based on simpler mechanisms such as local enhancement. That simple associations based on seeing food being exchanged can lead to preferences for some location over the other had been shown in a recent study with capuchin monkeys, Cebus apella (Brosnan \& de Waal, 2009). Here subjects were trained to return tokens to one of 
two human experimenters. One of the experimenters reliably rewarded the subject with food after s/he had received the token; the other one was an unreliable exchanger (i.e. failed to give a reward after receiving the token). In one condition, the reliable and unreliable exchanger switched positions after the subject had received the token. Analyses showed that the capuchins returned the token to the location where they had received it previously, but not to the reliable exchanger. They also failed to choose the reliable experimenter after watching interactions between another capuchin monkey and the two experimenters. The findings suggest that simpler mechanisms, such as a bias for spatial location, could be involved in this kind of experiment and potentially underlie some of the positive results reported so far (Brosnan \& de Waal, 2009). Indeed, location biases have also been found in dogs in different experimental set-ups, most often showing a preference for the location where they last saw a reward (Doré et al., 1996, Fiset and LeBlanc, 2007, Fiset and Plourde, 2013, Miller et al., 2009 and Rooijakkers et al., 2009).

In the current experiments, we tried to find out whether the dogs' performance in a social-eavesdropping paradigm is potentially influenced by a location bias. For this reason, we performed two experiments following the methodology of Marshall-Pescini, Passalacqua, et al. (2011). In the first experiment, an unknown stranger played the part of the beggar whereas this role was filled by the owner in the second experiment. Critically, we added a side control condition to both experiments. In this side control condition the two donors (generous versus selfish) swapped positions in half of the trials after the demonstrations but before the subjects were free to choose. With this additional condition we can control for the influence of local cues provided during the demonstrations (e.g. food transfer on only one side).

\section{Experiment 1}

In this experiment we assessed whether dogs use information about two unknown experimenters (the donors) after having witnessed interactions between those two donors and a third person (the beggar). They observed the unknown beggar begging from the two donors, with the 'generous' donor giving food to the beggar and the 'selfish' donor withholding the food. The prediction was that if dogs are able to use the indirect information about the food-sharing behaviour of the two donors, they should preferentially approach the 'generous' donor first and/or interact with her for longer even if the two donors swap positions before the dogs choose.

\section{Methods}

Subjects

Forty-eight dogs, 24 males and 24 females, living as pets with their owners participated in this experiment. Ten additional dogs had to be excluded for several reasons (four subjects never chose any of the experimenters, four subjects were uncomfortable in the test situation, one subject snatched a piece of food from one experimenter during the first test trial and one subject was not videotaped because of technical 
problems). For more detailed information about subjects in experiment 1, see Appendix Table A1. Only dogs older than 1 year (mean age \pm SD $=5.8 \pm 3.1$ years) and unfamiliar with both experimenters were selected from a database of owners who had volunteered to participate in this type of behavioural study. No breeds were excluded. The experiment was conducted in a room dedicated to dog studies. The owners of the dogs were present throughout the procedure.

\section{Procedure}

Set-up and experimental design

The experiment took place in a small empty room $(2.90 \times 3.80 \mathrm{~m})$. The two female experimenters were seated $2.5 \mathrm{~m}$ across from each other. The subject was placed perpendicular to and equidistant from the experimenters (1.5 m, see Fig. 1) and was held by its owner. A curtain was placed in front of the subject (distance $50 \mathrm{~cm}$ ). The whole procedure was videotaped by a wide-angle video camera, which was positioned on a tripod located next to the door (Fig. 1).

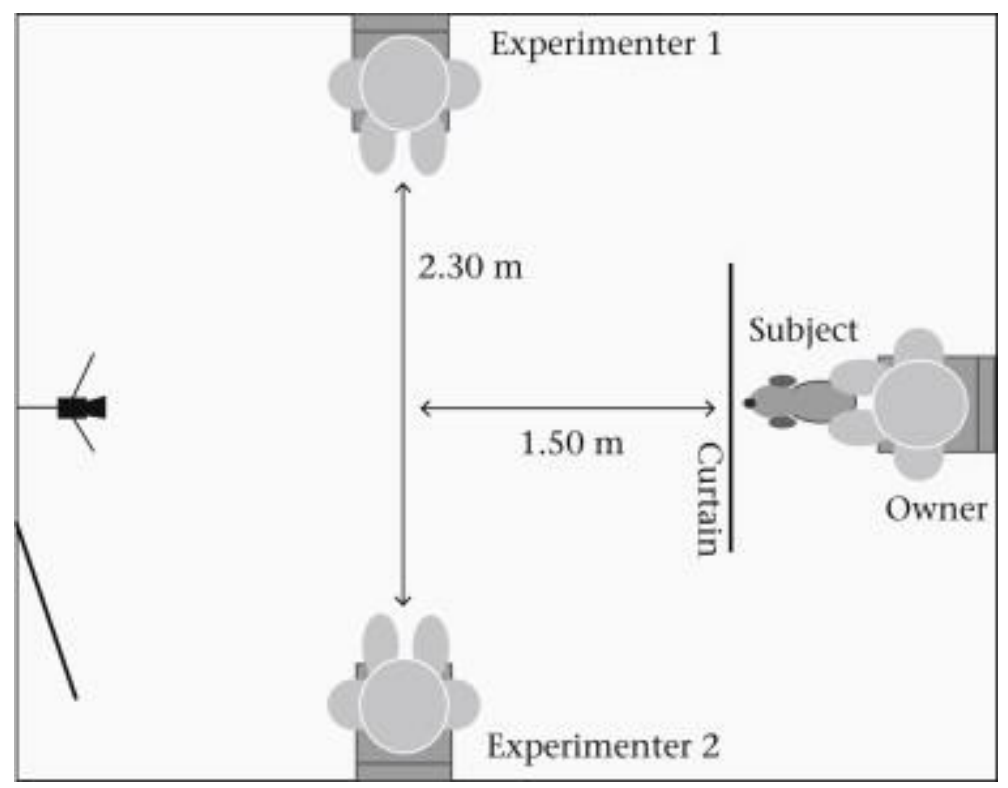

Figure 1.

Set-up of the experiments (adapted from Marshall-Pescini, Passalacqua, et al., (2011).

Before the test started, the experimenter (M.N.) explained the procedure to the owner, while the dog was allowed to move around the room freely. Following Marshall-Pescini, Passalacqua, et al. (2011), each trial consisted of two parts: observation phase and test phase. Each dog participated in four complete trials (observation + test), with the positions of the experimenters counterbalanced within and across subjects. Half of the dogs experienced M.N. being generous and half of the dogs experienced M.N. being selfish (mirrored by the second experimenter K.E.). Each observation phase lasted approximately 50 s, and each 
test phase lasted $20 \mathrm{~s}$. The subjects were randomly assigned to the experimental or the side control condition (see Appendix Table A1).

\section{Observation phase}

In the experimental condition, we followed the procedure of the experimental group in Marshall-Pescini, Passalacqua, et al. (2011). The owner was asked to sit down on the allocated location and to hold his/her dog between his/her legs, not to interact with the dog and to remain seated. The two female experimenters entered the room, each holding a bowl with two separate compartments. One of the compartments contained sausages, which were strong-smelling in order to attract the dog's attention, while the other contained cereals, which were eaten by the experimenters and by the human beggar during the demonstration. The two experimenters simultaneously approached the subject and presented the contents of the bowls. After the subject had inspected the bowls, both experimenters sat in their allocated positions, looked straight ahead, and simultaneously started eating the cereals. Now, the beggar entered the room and started to beg from one of the experimenters by kneeling down beside her and peering inside the box while tapping her arm. The experimenters responded according to their role. The generous donor said 'Nimms dir' (German for 'Have it') using a friendly voice while placing one piece of food in the beggar's mouth. The selfish donor said 'Nein' (German for 'No') in a firm voice and gestured the beggar away by flicking her hand out. The beggar alternated between the two researchers a total of six times. The sequence was semirandomized with no more than two interactions with the same donor in a row. The beggar spent the same amount of time next to each experimenter. Both donors ate the same amount of food throughout the observation phase. After the demonstrations, the beggar left the test room and the test phase began.

The procedure of the observation phase for the side control was almost the same as in the experimental group. The only difference was that the beggar did not leave the room immediately after the demonstrations. Instead, she approached the curtain in front of the dog and closed it. When the curtain was closed, the two experimenters swapped places in half of the trials (see Video 1 in the Supplementary material). For the other half of trials, both donors stood up, went half of the distance and went back to their previous position (see Video 1). The order of swap/stay was counterbalanced across subjects and randomized within subjects. After both donors were seated again, the beggar opened the curtain and left the room. The owner then released the dog.

\section{Test phase}

The procedure in the test phase was the same for both conditions and followed the procedure of MarshallPescini, Passalacqua, et al. (2011). The owner released the dog as soon as the door was closed behind the beggar. The owner was allowed to give a short free prompt once but not to gesture in a certain direction. 
The subject was free to move about within the room for $20 \mathrm{~s}$. During this time, both experimenters remained seated with the bowls on their laps. They looked down at the bowl with a neutral facial expression and never looked at or interacted with the subject. Only when the dog became intrusive, tried to jump up or snatch food out of the bowl did the researcher hold the bowl out of the dog's reach until s/he was back on the ground. Subjects never received food during the test trial. After $20 \mathrm{~s}$ the third person (beggar) knocked on the door to signal that the test trial was over. The subject left the test room together with the owner. Outside the test room, the owner gave a slice of sausage to the dog in order to maintain the subject's motivation.

After the donors were seated in their respective positions for the next trial (the position of the donors was counterbalanced between trials), the owner was asked to re-enter the room and take a seat at the predetermined location. Once the owner and the dog had settled down, the second observation phase began. Every dog underwent four observation phases, each of which was followed by a test phase.

Analysis and coding

Following Marshall-Pescini, Passalacqua, et al. (2011), we measured the gazing behaviour during the observation phase as well as certain behaviours during the test phase. All coding was done with INTERACT9 (http://www.mangold-international.com/software/interact). During the observation phase, we coded percentages based on towards which person the subject oriented his/her head and/or body as (1) gaze at generous donor, (2) gaze at selfish donor, (3) gaze at beggar, (4) gaze at owner and (5) gaze other. When the beggar kneeled next to one of the experimenters, it was not always possible to discriminate at whom the subject was looking. In such cases the dog was said to be focusing on the donor who was closest to the beggar.

During the test phase, we measured the dog's first approaches and the duration of interaction with each person. For first approaches, we coded the person the dog approached first, stopped within arm's length from and in front of whom they adopted a static position (standing, sitting, lying) while being oriented towards that person, or the first person with whom the dog interacted directly (i.e. physically): (1) approach generous donor, (2) approach selfish donor, (3) ambiguous approach. An approach was considered to be ambiguous if the dog moved around in the room or adopted a static position without meeting the other criteria. Furthermore, we coded the frequency and duration of the subject's interactions with a specific person: (1) interaction with generous donor, (2) interaction with selfish donor, (3) interaction with owner, (4) other behaviour and (5) out of sight. The definition for an interaction was the same as for the first approach: remaining stationary within arm's length with their body oriented towards the person or interacting in a more direct manner by establishing physical contact. 
A second coder, unaware of the purpose of the study, coded $20 \%$ of the gazing behaviour during the observation phase and interaction behaviour during test trials for reliability purposes. Reliability for gazing behaviour during the observation phase was moderate: Spearman correlation: gaze duration at generous donor: $r_{S}=0.764, N=40, P<0.001$; gaze duration at selfish donor: $r_{S}=0.733, N=40, P<0.001$. Reliability agreement reached a high level for the interaction duration: Spearman correlation: generous donor: $r_{\mathrm{S}}=$ $0.965, N=40, P<0.001$; selfish donor: $r_{\mathrm{S}}=0.984, N=40, P<0.001$. Reliability analyses for the first choices reached an excellent level (Cohen's Kappa $=0.962, N=40, P<0.001$ ).

We analysed dogs' gaze preference (generous versus selfish donor) by calculating an index of the gazing durations: generous/(generous + selfish) $\times 100$. First approaches were analysed within groups (binomial test) and between groups (Fisher's exact test). For the analysis of the interaction durations, we calculated the percentage of trial time the dog spent interacting with the generous and the selfish donor for the first trial and over all trials. These data were compared within each group (paired samples $t$ test). All statistical tests were two tailed and the alpha level was set to 0.05 .

To test whether the probability of subjects choosing the generous donor first was influenced by the side manipulation in the side control condition (side same versus side change) we ran a generalized linear mixed model (Baayen, 2008) with binomial error structure and logit link function. In the full model, we included the side as a fixed effect and subject identity as a random intercept term $(N=96$, number of subjects $=24)$. To control for the effect of trial number, it was included as an additional fixed effect as well as the interaction between side and trial number. As an overall test of the significance of condition, we ran a likelihood ratio test (Dobson, 2002) comparing the full model as described above with the null model, which lacked the factor side and its interaction with trial number (Forstmeier \& Schielzeth, 2011). To test the significance of the interaction between side and trial number, we ran a likelihood ratio test comparing the full model with a reduced model lacking this interaction only.

To test whether the proportion of time dogs spent close to the generous experimenter was influenced by the side manipulation (change side versus same side) we used a generalized linear mixed model (Baayen, 2008). The model was implemented with the number of frames in the video which the dogs spent close to the generous and the other donor, respectively, and fitted with binomial error structure and logit link function. We included the predictor side as a fixed effect and subject as a random effect. Again, trial was included as a further fixed effect.

\section{Results}

Dogs preferentially looked at the 'generous' donor in the observation phase of both conditions (experimental: $60.2 \%, t_{23}=8.963, P<0.001$; side control: $60.7 \%, t_{23}=11.273, P<0.001$ ) but they did not 
differ in the looking preferences between the two conditions $\left(t_{46}=-0.360, P=0.720\right)$. The analysis of first approaches in the experimental group revealed no preference for choosing a specific experimenter (see Appendix Table A2). However, subjects in this group tended to switch their preference in the second trial compared to the first trial (Fisher's exact: $P=0.056$ ). Regarding the duration of interactions, dogs did not interact preferentially with the generous donor over trial time or in the first trial (see Appendix Table A3).

In the side control, we found no evidence that dogs approached the generous donor first in trial 1, trial 2 and trial 4, but in the third trial, dogs preferred to approach the selfish donor first (see Appendix Table A2). First choices differed significantly between the second and the third trial $(P=0.015)$. Dogs did not interact longer with the generous donor over trial time or in the first trial (see Appendix Table A3). Additionally, we compared the interaction durations of the experimental condition with those of the same side trials of the side control to assess the potential impact of the curtain used in the side control condition, but found none (independent samples $t$ test: generous: $t_{46}=-0.543, P=0.589$; selfish: $t_{46}=0.181, P=0.858$ ).

Investigating the influence of the side manipulation on the first choice behaviour, we found that the full model was significant compared to the null model (likelihood ratio test: View the MathML source, $P=$ 0.038). After the removal of the nonsignificant interaction between condition and trial number (View the MathML source, $P=0.89$ ) it appeared that the probability of choosing the generous donor first was clearly influenced by the side, with dogs choosing the generous donor more often when she remained at the same side (estimate $\pm \mathrm{SE}=1.19 \pm 0.48, P=0.014$ ). The trial number had no effect on the first choice behaviour $(-0.34 \pm 0.21, P=0.108)$.

We found that side also had a clearly significant effect (see Appendix Table A4) on the interaction durations whereby the proportion of time spent close to the generous experimenter was higher when the experimenter stayed at the same side than after a side change (Fig. 2). Furthermore, the proportion of time spent next to the generous donor declined over trials (see Appendix Table A4). 


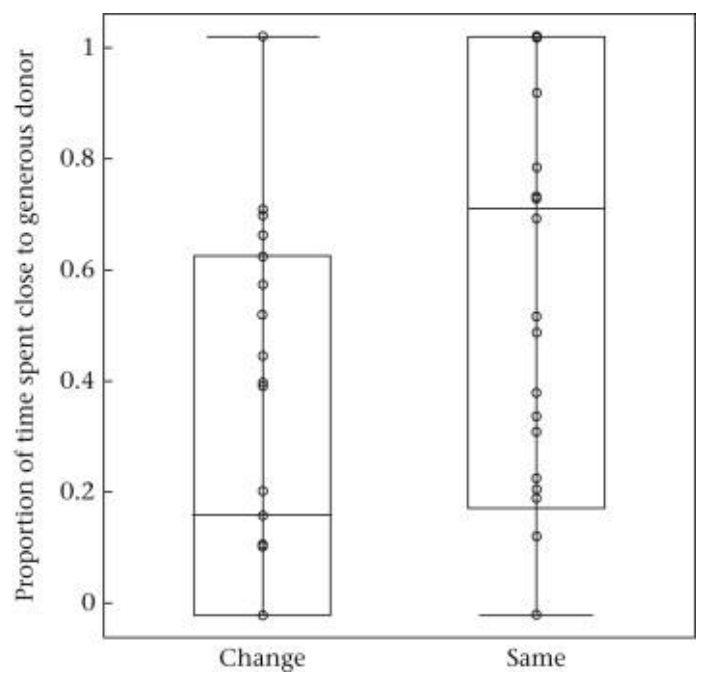

Figure 2.

Proportion of time spent close to the generous donor in the two experimental conditions. The thick horizontal lines show the median, the boxes depict the quartiles and the vertical lines show the percentiles (2.5 and 97.5\%). The circles represent the single subjects.

Comparison with Marshall-Pescini, Passalacqua, et al., (2011)

Comparison of our first choice data for the experimental condition with the results found by MarshallPescini, Passalacqua, et al. (2011) revealed that the two sets of data were not different in the first trial (Fisher's exact test: $P=0.29)$ but differed significantly in the second $(P=0.02)$ and third trials $(P=0.008)$.

Comparison of our first choice data from the side control (generous versus selfish) with those of the experimental group in Marshall-Pescini, Passalacqua, et al. (2011) revealed significant differences in trials 1 $(P=0.009)$ and $3(P<0.001)$ but not in trial $2(P=0.3)$.

\section{Discussion}

The results of the experiment presented here showed no evidence that dogs consider humans' foodsharing tendencies when choosing between two unknown persons after witnessing them interacting with a third unknown person. The subjects in our experimental group showed no preference for the 'generous' experimenter, either in first approach or in interaction times. However, they switched the preference in their approaching behaviour after the first trial (towards the selfish experimenter), most likely because the 'generous' experimenter did not give food during the first trial and they wanted to check whether the other person might give them some food. This is a clear indication that they had no problems in discriminating between the two persons and we can exclude this point as a potential limitation. In the side control condition, dogs switched their approach preference after the second trial, which resulted in a preferential approach towards the 'selfish' experimenter in the third test trial. Regarding the interaction times, we found no significance differences in the experimental condition. The results of the side control revealed 
that dogs chose the 'generous' donor first more often if she stayed at the same side as in the demonstration compared to the trials when she changed side. We found the same pattern for the interaction times. Compared with the 'selfish' donor, the dogs spent more time close to the 'generous' donor if she remained at the same side than when she changed side (see Fig. 2). This finding aligns with the outcome of Brosnan and de Waal (2009), who found a strong location bias in a similar setting.

Although we tried to follow the procedure of the experimental group in Marshall-Pescini, Passalacqua, et al. (2011) as much as possible, we were unable to replicate their results. Instead, we found some indication that dogs have a strong bias for spatial location. Potentially, the interactions of the experimenters with the unknown beggar were simply not relevant enough for the dogs to pay much attention to the actual interactions of the donors, although they were generally very attentive. Subsequently, we therefore conducted a second experiment with almost the same method as in experiment 1 , but this time the subject's owner, instead of an unknown person, begged from the two different experimenters to increase the salience of the interactions.

\section{Experiment 2}

In experiment 2, we tested whether dogs use the information about the typical behaviour of the two donors (same as in experiment 1) after having witnessed several interactions between the donors and their owner. The important role of the owner might enhance the salience of the donors' roles so that the dogs potentially consider the experimenters' behaviour as more relevant.

\section{Methods}

\section{Subjects}

Forty-eight dogs, 24 males and 24 females, living as pets with their owners participated in this experiment. Twelve additional dogs had to be excluded (10 subjects were excluded because their owner made a procedural mistake, one subject never chose any of the experimenters, one subject was uncomfortable in the test situation). For more detailed information about subjects in experiment 2, see Appendix Table A1. All dogs were older than 1 year (mean $4.8 \pm 2.5$ years) and unfamiliar with both experimenters.

\section{Procedure}

Set-up and experimental design

This experiment took place in the same room as experiment 1 . The set-up was also the same as in experiment 1 (see Fig. 1). The only difference was that the dog was not held by the owner but instead tethered to a hook on the ground. Prior to testing, the owner was given the instructions by the experimenter. The owners were not aware of the hypotheses and the purpose of the study. During this time, the dog was allowed to move around the room freely. 


\section{Observation phase}

The procedure of the experimental observation phase in this experiment resembled the procedure in experiment 1, although there was one notable difference. The beggar in this experiment was not an unknown person but the owner of the subject.

In contrast to the first experiment, the owner did not hold the dog during the observation. Instead, the helper (whom the dog did not know) fixed the subject at the allocated position. The demonstrations largely resembled those in experiment 1 , except for one difference. The 'generous' donor offered a piece of cereal by holding the bowl out to the owner (instead of placing a piece of cereal in the beggar's mouth as in experiment 1). The owner took one piece of cereal and ate it. As in experiment 1 , the owner alternated between the two researchers a total of six times. Both donors ate the same amount of food during the observation phase. After the demonstrations, the owner was instructed to stand behind the dog in an upright position and to position his/her dog equidistant between the two donors. After a few seconds the owner released his/her dog.

The demonstrations in the side control were exactly the same as in the experimental group. However, in contrast to the experimental group the owner did not release the dog after placing him/her in the right position. Instead s/he positioned the dog equidistant to the donors and held her by the collar. The helper (whom the dog did not know) entered the room, approached the curtain in front of the dog and closed it. When the curtain was closed, the two experimenters swapped places in half of the trials. After both donors were seated again, the helper opened the curtain and left the room. After the door was closed behind the helper, the owner released his/her dog. For a video clip of this condition, see Video 2 in the Supplementary material.

Test phase

The procedure of the test phase was the same for both conditions and was the same as that of experiment 1.

Analysis and coding

As in experiment 1 , we coded the gazing behaviour during the observation phase and certain behaviours during the test phase, using INTERACT9. We used the same definitions for the behaviours as in experiment 1.

For reliability purposes, a second coder (same as in experiment 1, unaware of the purpose of the study) coded $20 \%$ of the gazing behaviour during the observation phase and interaction behaviour during test 
trials. Reliability analyses for gazing behaviour and interaction behaviour reached high agreement in all measures: Spearman correlation: gaze at generous donor: $r_{\mathrm{S}}=0.929, N=40, P<0.001$; gaze at selfish donor: $r_{s}=0.913, N=40, P<0.001$; interact with generous donor: $r_{s}=0.982, N=40, P<0.001$; interact with selfish donor: $r_{S}=0.992, N=40, P<0.001$. Reliability for the first choices was excellent (Cohen's Kappa $=1, N=40, P<0.001)$.

We ran exactly the same analyses as in experiment 1.

Results

During the observation phase, the subjects preferred to look at the 'generous' experimenter in both conditions (experimental: $61.0 \%, t_{23}=12.497, P<0.001$; side control: $60.1 \%, t_{23}=11.225, P<0.001$ ) and they did not differ in the looking preferences between the two conditions $\left(t_{46}=0.681, P=0.499\right)$.

In the experimental group of this experiment, we replicated the results found by Marshall-Pescini, Passalacqua, et al. (2011) in some aspects: Analysing the first approaches of the experimental group, we found a preference for approaching the generous donor in trial 4 and a nonsignificant trend towards approaching the generous donor in trial 2 but no preference in trials 1 and 3 (see Appendix Table A5). The analyses of the interaction durations revealed that, overall, across all four trials, subjects in the experimental group interacted preferentially with the generous experimenter, but we could not find any significant preference when analysing the interaction data of the first trial separately (see Appendix Table A6).

In the side control, the analyses of the first approaches revealed no preference in approach in all four trials (see Appendix Table A5). Additionally, we found no evidence for subjects interacting preferentially with the generous donor when controlling for the spatial cue in the overall interaction times or in first trial analysis (see Appendix Table A6). We found no differences in the interaction times between the experimental condition and the same side trials of the side control condition (generous: $t_{46}=-0.494, P=0.624$; selfish: $t_{46}$ $=-1.163, P=0.251)$, indicating no impact of the curtain.

We could not detect an influence of the side on the first choice behaviour (likelihood ratio test: $\chi_{2}^{2}=1.63$, $P=0.441$ ), but again, we found that side had a clearly significant effect (see Appendix Table A7) on the proportion of time spent close to the 'generous' experimenter. Furthermore, the proportion of time spent next to the 'generous' donor increased over trials (see Appendix Table A7). 


\section{Discussion}

In the experimental condition of our second experiment, dogs preferred to interact with the generous donor, approached the generous donor in one of four trials and tended to approach her in another trial. This means that having the subject's owner as the beggar we largely replicated the results found by Marshall-Pescini, Passalacqua, et al. (2011).

In the side control, we found no significant preference for either person, either in the interaction durations or in the first choices. Additionally, the change of side influenced the proportion of time spent close to the 'generous' donor, showing again that the dogs did not rely on the information about the experimenters' behaviour they should have gained during the demonstration.

As in experiment 1, we found no indications that the subjects preferred the 'generous' experimenter when we controlled for potential bias for spatial location, which indicates that they did not use the information about the experimenters' food-sharing tendencies flexibly.

\section{General discussion}

In our study we found no support for the hypothesis that dogs are able to assess humans' food-sharing tendencies after having witnessed interactions of a food-sharing experimenter versus food-withholding experimenter with a third person, the beggar. Instead, we found indications of an alternative explanation for the dogs' behaviour in this setting: It was influenced by the side, which was emphasized during the demonstrations.

In the experimental conditions (i.e. when not controlling for potential spatial associations) we found an effect only when the owner played the role of the beggar, which suggests that the interactions were more salient if both experimenters interacted with the owner instead of an unknown person. Owner-stranger effects were also found in other studies. For example, Elgier, Jakovcevic, Mustaca, and Bentosela (2009) found that dogs were faster in a reversal learning task but slower in an extinction task when the owner, and not an unknown experimenter, pointed to one of two containers. Comparing two studies on a manipulative learning task, dogs learned better if the owner demonstrated the action (Kubinyi, Topal, Miklósi, \& Csányi, 2003) than if an unknown experimenter demonstrated how to reach the reward (Range et al., 2009). By contrast, owner and stranger were equally effective as demonstrators in a detour task (Pongrácz et al., 2001). Furthermore, an unknown friendly person does not influence dogs' food choice behaviour less than the owner (Marshall-Pescini, Prato-Previde, \& Valsecchi, 2011). Another possibility for the difference in the results is the difference between the demonstrations in the two experiments. In experiment 1, the 'generous' donor placed the piece of food in the beggar's mouth, whereas in experiment 2 the 'generous' donor held the bowl with the food out so that the beggar (=owner) could reach for one on her own. So the 
dog saw the hand-to-mouth movement performed by two different persons in experiment 2 (and only one person in experiment 1). Marshall-Pescini, Passalacqua, Miletto Petrazzini, Valsecchi, and Prato-Previde (2012) have shown that this particular action is a very salient cue for dogs and can even induce counterproductive choices in a food choice task. So it is possible that they preferred the side where the owner performed the hand-to-mouth movement.

In the side control conditions of both experiments, we found no evidence that dogs preferred the 'generous' donor after she had changed her position in half of the trials. It could be that the subjects simply were not able to distinguish between the two persons after they had switched sides and therefore went to the side where they noticed the food transfer, as this was the only cue they could rely on. While we cannot totally rule out this alternative explanation, it is none the less unlikely as dogs are able to discriminate between two human individuals by facial cues (Racca et al., 2010) or scent (Schoon, 1997) alone and both traits were available to them during the whole procedure. Additionally, subjects switched preference in approaching the experimenters in the side control of experiment 1 , which indicates that they are able to differentiate between the two persons. However, this alternative explanation would also not support the proposed hypothesis that dogs are able to assess humans' food-sharing tendencies (Kundey et al., 2010 and Marshall-Pescini, Passalacqua, et al., 2011), as recognition of the persons would be a basic prerequisite.

Also, the delay between the demonstrations and the test trial in the side control caused by the drawn curtain during side changes could have influenced the dogs' choice behaviour. Specifically, the subjects in the side control condition may simply have forgotten which person gave the food to the beggar. As the delay lasted only approximately $9 \mathrm{~s}$, it is unlikely that dogs could not recall the interactions of the donors with the beggar. A study on working memory in dogs showed no decrease in performance in an object permanence task after such a short retention interval, which suggests that dogs maintain an active representation of the situation during a short delay (Fiset, Beaulieu, \& Landry, 2003). Even more relevant, dogs are able to memorize information about 'what' and 'where' for more than 10 min (Fujita et al., 2012). More importantly, the comparison of the trials of the experimental condition with those trials of the side control condition in which the donors did not swap sides revealed no differences in both experiments. As the use of the curtain is the only procedural difference between these trials we can conclude that it has no impact on the results.

Another possibility is that subjects understood the firm 'no' from the 'selfish' experimenter as a prohibition to go to that location. This alternative explanation is unlikely for two reasons. Marshall-Pescini, Passalacqua, et al. (2011) conducted a ghost control condition, in which the experimenters performed their actions (the same as in our study) without a beggar being present. If they had understood the selfish gesture as prohibition, then they should have preferred the generous donor but dogs in that condition 
chose randomly between the two experimenters. Additionally, dogs do not understand prohibiting motives in a competitive situation of an object choice task (Pettersson, Kaminski, Herrmann, \& Tomasello, 2011).

Although all subjects were very attentive throughout the demonstrations in general, it is also possible that they did not pay so much attention to the actual interactions, or the experimenters' behaviour, as they were not intended for them (i.e. no ostensive cues were directed towards the dog; see Kaminski et al., 2012).

In sum, we found no confirmation of dogs' ability to use information gathered via social eavesdropping. In contrast, we found some indication that dogs' behaviour was driven by simpler, less cognitive mechanisms. It is possible that they simply went to the side at which they preferentially looked during the demonstrations (which is the side where the 'generous' donor was seated during the observation phase). This hypothesis might also apply to the results of Marshall-Pescini, Passalacqua, et al. (2011), as the subjects only preferred the 'generous' donor in the two conditions in which they also looked preferentially to her during the demonstration phase. In those two conditions, as well as in our experiments, the 'generous' donor uttered acoustic signals using a friendly and cheerful voice (as opposed to the 'selfish' donor), which potentially could have led to a social enhancement of this location, causing longer looking times and resulting in a preference for that side. Other studies also show that the human voice is a very salient cue for dogs. For example, they solve a detour task more effectively if the human demonstrator attracts the dogs' attention verbally (Pongrácz, Miklósi, Timár-Geng, \& Csányi, 2004).

The fact that the dogs saw the beggar eating on the 'generous' side could have led to a bias towards this location. Palameta and Lefebvre (1985) found that pigeons, Columba livia, solved a problem (piercing a piece of paper covering a food box) faster and more efficiently if they saw a model piercing the paper through a red dot and eating food from the box than when they saw the model only piercing the cover and not eating. Presumably, the subjects in the piercing-eating condition associated the red dot on the paper with food, which helped them to solve the problem ( Heyes, 1994). Hoppitt and Laland (2008) also consider a similar explanation in food patch assessment as individuals watch conspecifics eating ( Coolen et al., 2003, McQuoid and Galef, 1993 and Templeton and Giraldeau, 1995). In line with this account, one could argue that the side where the 'generous' donor sat during the observations is evaluated as a food patch of good quality (i.e. more food is available) whereas on the side where the 'selfish' donor sat less food is available.

In general, some such social enhancement of side almost certainly played a role in our study, as the 'generous' donor used a friendly tone of voice and the food was also handled more on the 'generous' side: two highly salient cues which could potentially induce a social bias (Marshall-Pescini et al., 2012). However, even more important was the fact that the beggar received a reward at the 'generous' location but not at 
the 'selfish' location. This means the dogs saw the beggar eating at the place where the 'generous' donor sat and they link this location with food. In contrast, the beggar did not eat while s/he was close to the 'selfish' experimenter. The fact that dogs did not use the information about experimenters' behaviour when no food was involved and no local cues were provided strengthens the assumption that simpler mechanisms potentially play a role in this kind of set-up (Nitzschner et al., 2012). Additionally, a recent study on a different aspect of dogs' social-eavesdropping abilities found similar results (Freidin, Putrino, D'Orazio, \& Bentosela, 2013). In that study, the distinguishing feature between the two donors was not their own behaviour but the behaviour of the approaching beggar who reacted differently to the donors. The beggar ate the offered cornflake from the 'positive' donor but rejected the cornflake from the 'negative' donor. The dogs in this condition developed a preference for the 'positive' donor. However, similar to our results, the dogs did not show any preference in two control conditions in which the donors changed sides or the donors were replaced by inanimate objects. The study we have presented is, therefore, the second one in which controlling for local cues did not lead to a replication of previous findings (Kundey et al., 2010 and Marshall-Pescini, Passalacqua, et al., 2011). We have no conclusive explanation why we failed to replicate the results found by Marshall-Pescini, Passalacqua, et al. (2011) in our experiment 1 although we tried to match their procedure as closely as possible. Potentially other factors, which we did not control for, such as training or breed, could have an impact on the results.

However, the results of this study suggest that dogs do not use information about people's food-sharing behaviour gained through eavesdropping flexibly as proposed in previous literature. Although these findings are not totally conclusive, they highlight the importance of controls for location biases in this kind of behavioural study.

\section{Acknowledgments}

We thank the dog owners, without whose support this work would not be possible. We also thank Kerstin Esau, Katrin Schumann, Marlen Hinz and Julia Steinbrueck for help with data collection and Anne Hertel for reliability coding. We thank Roger Mundry and Colleen Stephens for statistical advice. We are very grateful to Sarah Marshall-Pescini for valuable comments on the manuscript. 